\title{
Les Archives
}

Ou l'éducation à la neutralité axiologique

Jean Baubérot

\section{(2) OpenEdition}

Journals

Édition électronique

URL : http://journals.openedition.org/assr/3814

DOI : 10.4000/assr.3814

ISSN : $1777-5825$

Éditeur

Éditions de l'EHESS

Édition imprimée

Date de publication : 1 décembre 2006

Pagination : $39-51$

ISBN : 2-7132-2124-2

ISSN : 0335-5985

Référence électronique

Jean Baubérot, "Les Archives », Archives de sciences sociales des religions [En ligne], 136 | octobre -

décembre 2006, mis en ligne le 02 janvier 2009, consulté le 20 avril 2019. URL : http://

journals.openedition.org/assr/3814 ; DOI : 10.4000/assr.3814 


\section{Jean Baubérot}

\section{Les Archives \\ Ou l'éducation à la neutralité axiologique}

Ne faisant pas partie de l'équipe fondatrice des Archives de sociologie des religions, mais de la génération qui en a reçu l'enseignement (transmission du charisme ?!), je voudrais apporter ici un modeste témoignage. Il ne me paraît pas inutile dans le cadre de ce numéro de souligner que le premier relais générationnel s'est effectué lors de la seconde moitié des années 1960 et de la première moitié des années 1970. Ce furent des années de contestations politique et religieuse intenses, où la sécularisation interne des institutions ecclésiales constituait la stratégie dominante des groupements religieux.

Ce constat renvoie à un contexte précis marqué par l'après-guerre d'Algérie, la guerre du Vietnam, la révolution culturelle chinoise et les guérillas en Amérique Latine, la guerre des Six Jours au Proche-Orient et ses suites, les crises étudiantes avec, notamment, en France l'effervescence de « Mai 68 » et l'essor de ce qui était appelé alors les « groupuscules ». Ensuite, ce sera (pour ne parler que de la France) la progressive mise en place de l'union de la gauche et la marche en avant vers une alternance démocratique, qui se réalisera en 1981, avec la victoire de François Mitterrand à l'élection présidentielle. L'avant et l'après "Mai 68 " fut donc l'époque où la jeunesse intellectuelle se précipitait pour aller voir les films de Godard dès leur sortie et suivre les cours (mais fallait-il encore parler de "cours » ?) de Lacan, Foucault, Barthes... Les étudiants dévoraient les ouvrages de Derrida, écoutaient presque religieusement Sartre haranguer les ouvriers de BoulogneBillancourt. Dans le christianisme, les grandes figures semblaient être celles qui proposaient un christianisme révolutionnaire, anti-impérialiste, une théologie de la Révolution liée à la théologie de la libération latino-américaine : l'équipe qui publiait la revue franciscaine Frères $d u$ Monde, le dominicain Jean Cardonnel, le professeur de la faculté protestante de théologie de Paris Georges Casalis. Selon eux, un propos, pour être légitime, devait obligatoirement être engagé, aller dans le sens d'une révolution politique anti-impérialiste. Tout discours se prétendant objectif cachait, en fait, des idées contre-révolutionnaires et devait être dénoncé comme tel. C'était enfin l'époque, on l'oublie trop aujourd'hui, où invoquer les "valeurs de la République " apparaissait le comble du slogan 
40 - Archives de SCIENCES Sociales des Religions

«réactionnaire »: la République était fondamentalement «bourgeoise", elle représentait le «vieux monde» à abattre pour pouvoir édifier un «monde nouveau ".

Un tel contexte aurait pu (aurait dû, pourrait-on presque écrire) éloigner les apprentis sociologues de l'équipe fondatrice, certes très diverse, mais ayant en commun la volonté de pratiquer la neutralité axiologique. Pourtant, il n'en fut rien et les chercheurs francophones ${ }^{1}$ en sociologie des religions, ou dans d'autres disciplines des sciences humaines, qu'ils appartiennent aujourd'hui au CEIFR, au GSRL, à d'autres laboratoires, les universitaires ou membres du CNRS qui aujourd'hui atteignent ou ont dépassé la soixantaine ont été nombreux à s'être trouvés profondément marqués par les Archives, à avoir été formés par les «ténors " de l'équipe de rédaction. Chacun peut préciser l'héritage scientifique reçu, et telle ou telle occasion a permis de le faire au moins partiellement ${ }^{2}$. Mais l'objectivation du propos ne permet pas d'examiner une question importante: comment s'est concrètement effectuée la transmission de l'idéal de scientificité et de la mise en œuvre de la neutralité axiologique, à une époque où l'engagement total apparaissait comme seul légitime, la mise en question du christianisme établi sur des bases "révolutionnaires " la seule manière non "aliénante " de continuer à s'en réclamer peu ou prou ou, du moins, de s'y intéresser ? Un témoignage délibérément subjectif, et comportant toutes les caractéristiques non scientifiques que Pierre Nora attribue à la mémoire, peut alors constituer un apport pas complètement inutile pour une analyse qui reste à faire ${ }^{3}$.

Ma rencontre avec les Archives de sociologie des religions s'effectua en deux temps. Le premier eut lieu avant "Mai 68 ", notamment dans le cadre de ma thèse de doctorat de troisième cycle (1964-1966) et se focalisa sur les écrits d'Émile-Guillaume Léonard qui représentait l'étude du protestantisme dans le groupe (où il resta une figure relativement marginale mais très estimée); le second, quelques années plus tard, entraîna ma progressive intégration dans le laboratoire qui était le principal éditeur des Archives, le Groupe de sociologie des religions, ainsi qu'une collaboration à la revue ${ }^{4}$.

1. Et pas seulement français, que l'on pense à l'influence des Archives dans d'autres pays européens, au Québec, au Mexique, en Amérique latine, etc.

2. Cf. par exemple, les journées d'hommage à Émile Poulat (22-23 octobre 1999) publiées par V. Zuber, Émile Poulat, un objet de science, le catholicisme, Paris, Bayard, 2001.

3. Et qui devra, naturellement, non seulement s'appuyer sur un ensemble de témoignages plus diversifiés, mais avoir d'autres sources d'investigations.

4. On l'aura compris : il ne s'agit pas ici de retracer un itinéraire personnel; il sera nécessaire cependant d'en donner les quelques éléments indispensables pour rendre compréhensible ce que les Archives m'ont apporté et, notamment, fait qui me semble extrêmement intéressant, pourquoi il s'est produit parfois un décalage de près de dix ans entre la parution de tel ou tel article et sa lecture approfondie. 


\section{É.-G. Léonard et les débuts de la sociologie du protestantisme}

Émile-Guillaume Léonard (1891-1961) tomba malade et mourut l'année universitaire même où je m'étais inscrit à ses cours donnés à l'École pratique des hautes études (1960-1961). Je ne pus donc bénéficier de son enseignement. Je me suis cependant intéressé à ses écrits. C'était un historien attentif à la démarche sociologique, à ce qu'il appelait « la sociologie historique et la sociologie psychologique » du protestantisme dont il fit une présentation dés le numéro 2 de la revue. Et il précisait : "dans un pays comme le nôtre [la France], le protestantisme est profondément produit social du passé ; d'autre part, la fidélité qu'il demande particulièrement à ses membres, comme religion minoritaire, y donne une place de première importance aux décisions et comportements individuels, plus ou moins conditionnés par le milieu ». Cela montrait une certaine réticence à une démarche sociologique en tant que telle dont Léonard craignait les «travaux de statistique et de sociographie ": "à compter les protestants, encore faudrait-il savoir où l'on établira la limite». Il concluait fort logiquement : "l'histoire sociale du protestantisme français [est la] base la plus sûre de sa sociologie religieuse ${ }^{5}$. Il reprit cette idée, quelques années plus tard, en indiquant que, même pour les périodes où «les événements sont à peu près connus, (...) tout "l'éclairage" est à revoir, en des études moins "historisantes" et moins "apologétiques" qui fassent droit aux "psychologies de classe", condition, selon lui, d'une véritable "sociologie du protestantisme" 6 ".

Cette histoire sociale, Léonard la construisait en refusant de traiter le protestantisme français de l'Ancien Régime, selon une formule qu'il affectionnait, « en petit garçon geignard persécuté par les méchants». Les historiens protestants insistaient sur " l'irrévocabilité » de l'Édit de Nantes pour mieux stigmatiser ceux qui avaient lutté contre son maintien. Il montre, dans différents travaux, que l'Édit constitue un point d'équilibre instable que chaque parti a tendance à considérer, dès sa promulgation, comme une mesure transitoire. Les protestants l'interprètent comme un point de départ et souhaitent que la paix religieuse qu'il instaure permette à leur confession de faire des progrès substantiels. Pourquoi s'indigner alors que le parti adverse (le parti dévot) fasse pression au contraire, pour une application restrictive de l'Édit?

En conséquence, c'est d'abord dans les faiblesses internes du protestantisme français que Léonard recherchait les raisons de sa chute, en effectuant une analyse fine des stratifications sociales de ce protestantisme et en mettant en relation les classes sociales et les options religieuses et idéologiques des protestants. Les rêves d'unionisme, les espoirs de " gallicanisme " induirent, dans la bourgeoisie

5. É.-G. LÉONARD, "Travaux de sociologie religieuse sur le protestantisme français ", ASR, 2, 1956, p. 43.

6. É.-G. LÉONARD, «Les conditions de la sociologie protestante en France », ASR, 8, 1959, p. $125-137$. 
protestante, un loyalisme très fort qui diminua la résistance aux pressions sociales qui précédèrent la révocation de l'Édit. Rupture profonde avec une histoire hagiographique ${ }^{7}$, la thèse de Léonard a le grand mérite d'indiquer que les mesures répressives ne constituent pas les seuls coups donnés au protestantisme français d'alors ${ }^{8}$. Première initiation à la neutralité axiologique !

Dès les premiers numéros des Archives, Léonard a donc porté le souci d'une réflexion et d'une pratique de sociologie historique du protestantisme. Historien de l'époque moderne, il s'est cependant aventuré dans la période contemporaine et a publié dans la revue une étude suggestive sur la stratification professionnelle des protestants français du dernier tiers du XIX ${ }^{\mathrm{e}}$ siècle, en étant attentif aux mandats électifs exercés par certains d'entre eux. La conclusion de ce travail, montre cependant, une réticence identique à celle déjà signalée devant des travaux qui majoreraient une "sociologie statistique du protestantisme » : " de la sociologie des masses nous sommes ainsi ${ }^{9}$ rejetés sur l'examen de situations et de milieux très délimités avec, comme aboutissement, l'étude des familles de telle ou telle région protestante, ou la participation protestante à tel ou tel corps de l'État. (...) L'histoire du protestantisme français, religion “de choix”, est acculée à l'individuel et au psychologique ${ }^{10}$.

Cette méfiance envers une sociologie quantitative, et aussi une certaine réserve envers les perspectives de Max Weber ${ }^{11}$, cette volonté de sauvegarder une image du protestantisme comme religion de la décision personnelle, est significative d'une époque où la sociologie du protestantisme émerge en France ${ }^{12}$. Léonard polémique implicitement contre une autre vision (plus sociographique) de la sociologie du protestantisme, qui s'exprime également à l'occasion dans les

7. Qui a cependant été, ensuite, jugée d'une sévérité excessive envers la bourgeoisie protestante du XVII ${ }^{\mathrm{e}}$ siècle. Ainsi, E. Labrousse montre qu'à partir du moment où la logique absolutiste était en marche, quelle que soit la stratégie mise en œuvre par les protestants, elle se retournait contre eux (Cf. "Une foi, une loi, un roi »? La révocation de l'Édit de Nantes, Paris-Genève, Payot-Labor et Fides, 1985).

8. É.-G. Léonard ne développa pas cette thèse dans les Archives, mais la revue suivit avec attention ses travaux, notamment en publiant des comptes rendus. Voir notamment celui que Jean SÉGUY consacre au tome II de l'Histoire générale du Protestantisme, in ASR, 13, 1962, p. $179-180$.

9. Par l'étude qu'il vient d'exposer.

10. É.-G. LÉOnARD, «Les professions dans la France Protestante en 1868 et 1893 », ASR, 5, 1958, p. 139.

11. La rédaction des Archives, en lui rendant hommage peu après sa mort, écrivait dans un éditorial signé GSR, après avoir rapporté ses réserves quant à la sociologie : "il a donné dans son Histoire Générale [du Protestantisme] un pénétrant exposé de son point de vue sur "Calvin créateur d'un type d'homme et de civilisation", qui ferait honneur à n'importe quel sociologue ", ASR, 14, 1962, p. 4.

12. R. Mehl venait d'organiser, en 1959, un colloque européen de sociologie du protestantisme à la faculté de théologie de Strasbourg, dont le $\mathrm{n}^{\circ} 8$ des $A S R$ publia les actes. Il créa dix ans plus tard le Centre de sociologie du protestantisme (cf. infra, note 20). 
Archives ${ }^{13}$, approche qu'il ne déprécie pas mais dont il a peur qu'elle devienne dominante. Cependant, son intérêt pour les dissidences religieuses (notamment le pentecôtisme brésilien ${ }^{14}$ ) rejoint celle de membres de la rédaction comme Henry Desroche ${ }^{15}$ et Jean Séguy ${ }^{16}$ ainsi que de collaborateurs extérieurs comme Bryan Wilson ${ }^{17}$ qui écrivirent régulièrement dans la revue.

En définitive, à la mort de Léonard, Gabriel Le Bras résumait la proximité et la distance de Léonard à l'égard de la sociologie en général et des Archives en particulier. Il discerne trois raisons pour lesquelles «ce savant historien » ne voulait point se dire sociologue : « il craignait de compromettre par l'attrait des généralités - types ou lois - la pureté de la trame historique; il redoutait de réduire le divin à l'humain ; peut-être mettait-il une certaine coquetterie à refuser la mode ». Nous trouvons là le double problème de la relation de la sociologie et de l'histoire et du dégagement de la sociologie de la religion de toute perspective théologique. Et Le Bras poursuit: "Il ne pouvait nous empêcher de le tenir pour un précieux allié, qui nous apportait (...) plus de sociologie qu'il ne voulait l'avouer ». Pour Le Bras, Léonard "esquissait (...) une sociologie historique du protestantisme ${ }^{18}$. Jean-Paul Willaime reprendra plus tard, dans la revue, les problèmes soulevés par Léonard : selon lui, ce dernier met l'accent sur une réelle difficulté : «comment étudier quantitativement une religion qui, constitutivement résiste à une objectivation statistique (encore plus si elle est minoritaire) ? ». Willaime propose de multiplier « les indicateurs de vitalité religieuse de façon à ne pas étudier le protestantisme à travers le filtre d'une conception trop étroite (éventuellement catholico-centrée) de l'excellence religieuse ${ }^{19}$.

Je ne développe pas davantage, car j'ai délibérément donné à ma contribution le caractère d'un témoignage et, si j'ai pris connaissance de certains articles autres que ceux de Léonard dans les Archives, sur le moment cela ne m'a pas particulièrement marqué. Pourquoi ? Essentiellement pour deux raisons qui témoignent toutes les deux d'une époque.

13. Cf. par exemple, P. LeSTRINGANT, « Remarques sur l'évolution sociologique du protestantisme français ", ASR, 8, 1959, p. 139-145 et F.G. DREYFuS, "Résultats d'une sociologie du protestantisme en France », ASR, 8, 1959, p. 147-157.

14. É.-G. LÉONARD, Illuminisme dans un Protestantisme de formation récente, Paris, 1952, tome LXV de la Bibliothèque de l'École pratique des hautes études, Sciences religieuses.

15. Par exemple, H. DesRoche, "Approches du non-conformisme français », ASR, 2, 1956, p. 45-54.

16. Par exemple, J. SÉGUY, "Les sectes d'origine protestante et le monde ouvrier français au XIX ${ }^{\mathrm{e}}$ siècle ", ASR, 6, 1958, p. 119-126; " Problèmes historiques et sociologiques actuels de l'anabaptisme ", ASR, 7, 1959, p. 105-115.

17. Par exemple, B. WiLson, "Apparition et persistance des sectes dans un milieu social en évolution ", ASR, 5, 1958, p. 140-150.

18. G. Le Bras, "Émile-G. Léonard ", ASR, 14, 1962, p. 5.

19. J.-P. Willaime, "Du protestantisme comme objet sociologique ", ASSR, 83, 1993, p. $163 \mathrm{sq}$. 
La première raison était qu'en un temps où n'existaient ni ordinateurs ni même photocopieuses, nous étions pourtant très fortement incités à réaliser en deux ans une thèse qui, par l'ampleur de la documentation (de multiples dépouillements d'archives, nationales et départementales, en ce qui me concernait) et la dimension (534 pages, une interligne pour ma part), était tout à fait équivalente aux thèses de doctorat actuelles (réalisée souvent en cinq ou six ans). Cela impliquait une forte "polarisation» sur son sujet de thèse et sa discipline : j'étais historien et, sur le plan scientifique, je lisais essentiellement des travaux d'historiens. Et pour ce qui concernait plus directement le protestantisme français, Roger Mehl, professeur à la faculté de théologie protestante, tenait, dans la revue, des propos trop prudents pour s'avérer très enthousiasmants. " Nous définirons, écrivait-il, la sociologie du Protestantisme comme la sociologie [des] groupes paroissiaux, dans lesquels s'exprime l'ecclésiologie propre à la Réforme ». Il souhaitait, cependant, aller plus loin que "l'actuelle sociologie » qui, selon lui, s'en tenait à la pure morphologie de cette communauté, à ses pratiques, à ses institutions pour chercher à «atteindre la vie interne de cette communauté ${ }^{20}$.

La seconde raison, c'est qu'en ces années précédant "Mai 68 ", beaucoup de temps se trouvait également consacré à préparer la Révolution, avec un grand R. Elle devait être à la fois, politique, culturelle, affective, sexuelle et religieuse. Pour la contestation politique, j'étais membre de l'Union des étudiants communistes (UEC) et plus exactement de la tendance dite "italienne » (influencée par Gramsci et l'évolution du PC italien), puis quand l'UEC fut reprise en main par le Parti communiste, membre du Centre révolutionnaire d'action et de recherche (CRIR), fondé par les «Italiens » de l'UEC. Il s'agissait de «refaire collectivement pour le $\mathrm{XX}^{\mathrm{e}}$ siècle ce que Marx et Engels avaient faits pour le XIX ${ }^{\mathrm{e}}$ siècle », rien de moins! Pour les autres aspects de cette révolution nécessaire, je fus directeur du Semeur, la revue du mouvement des étudiants protestants, qui proposait un abonnement à prix réduit pour "les couples tentant l'union libre ${ }^{21}$, puis après la crise du mouvement, directeur de la revue Herytem, critique politique de la vie quotidienne.

20. R. MEHL, "Significations, possibilités et limites de la sociologie du protestantisme ", ASR, 14, 1962, p. 9, 16. Voir également «Modifications dans la structure et le comportement des Églises protestantes de France à la suite du mouvement œcuménique ", ASR, 22, 1966, p. 81-88. R. Mehl inaugura, en 1964, un cours de sociologie du protestantisme à la faculté de théologie protestante de Strasbourg et créa, cinq ans plus tard, un Centre de sociologie du protestantisme dans cette même faculté (voir la présentation qu'il en fait dans les ASR, 30, 1970, p. 121-122).

21. On a de la peine à imaginer aujourd'hui le bruit et la fureur que provoquèrent les articles du Semeur dans ce domaine. Il faut se rappeler que la contraception fut interdite en France jusqu'à la loi Neuwirth (1967), que celles que l'on qualifiait de "filles mères » étaient l'objet d'une forte réprobation sociale, et que les «événements " de "Mai 68 » commencèrent par la contestation par des étudiants de l'interdiction de se rendre dans les chambres des étudiants de l'autre sexe, en cité universitaire. 
Il est sans doute étonnant de penser que certaines des préoccupations fortes des Archives ne m'aient pas intéressé alors. Il en fut pourtant ainsi et peut-être, paradoxalement, le décryptage de l'athéisme marxiste et des utopies messianistes qu'entreprenait Henri Desroche ${ }^{22}$, pour prendre un exemple, effectuait trop (indirectement) une analyse critique de la situation vécue pour être compris et admis. C'est une hypothèse. Par ailleurs, ma vie était soigneusement cloisonnée entre la préparation d'une thèse que mon directeur voulait rigoureuse et obéissant à des méthodes classiques en histoire (schématiquement, celles que l'on trouvait à la Revue Historique) et le reste de mon existence, tournée vers l'espérance d'une autre société. C'était, d'ailleurs, le contrat que m'avait fixé ce directeur, Daniel Robert ${ }^{23}$, un homme de droite très libéral qui m'avait prévenu qu'il se montrerait d'autant plus indifférent à ma mauvaise réputation, qu'il n'aurait rien à redire au travail "scientifique » que je lui remettrais ${ }^{24}$. Légitimée par le fait que le guérillero urbain travaillait normalement la journée, en se fondant dans la masse, afin de pouvoir participer, la nuit tombée, à une embuscade qui portait des coups au " pouvoir bourgeois ", cette double vie permettait de satisfaire à la fois à la passion de l'histoire et à la passion de la révolution ${ }^{25}$.

\section{La question de la " neutralité axiologique"}

La véritable rencontre des Archives eut donc lieu, pour ce qui me concerne, plus tard, dans la suite immédiate de " Mai 68 », et pour une raison sociologiquement intéressante : la routinisation de l'effervescence révolutionnaire. Si pour les jeunes étudiants, Mai 68 fut un point de départ, certains autres, plus âgé de quelques années, l'ont plutôt vécu très vite comme le feu d'artifice qui avait clôt

22. H. Desroche, "Athéisme et socialisme dans le marxisme classique : K. Marx-F. Engels ", ASR, 10, 1960, p. 71-108; "Genèse et structure du Nouveau Christianisme saint-simonien ", ASR, 26, 1968, p. 27-54.

23. D. Robert, successeur d'É.-G. Léonard à l'EPHE, ne collabora pas aux Archives, ceci indiqué, il manifesta " une ouverture à la sociologie », selon l'expression utilisée par J.-P. WILlaime, art. cité, ASSR, 83, 1993, p. 164.

24. D. Robert a toujours tenu ce contrat, même quand, en Mai 68 et ensuite, la contestation effectuée atteignait l'EPHE elle-même. Je lui en ai été d'autant plus reconnaissant.

25. Un exemple de cette prose légitimatrice : "Les forces au pouvoir sont tellement omnipotentes qu'elle aliènent même toute opposition. (...) Nous ne sommes pas des révolutionnaires professionnels. Nous vivons à peu près (en nous accrochant à cet "à peu près” dérisoire) comme tout le monde puisque nous y sommes bien obligés. Ce faisant nous participons au pouvoir et sommes parmi nos “ennemis” (...) Mais il n'est pas possible de vivre comme si la révolution était déjà accomplie. Il n'existe pas de “zones libérées” ou même libérables à brève échéance [même si...] dans l'éros et dans le logos nous pouvons parfois pressentir un peu quelle serait l'existence pour laquelle nous nous battons. Seulement de temps à autre quelques "échauffourées" sont possibles. Il nous arrive donc d'être de petits maquisards, un peu peureux, remettant vite l'uniforme des anonymes pour pouvoir feindre un air d'innocence face aux inquisiteurs et autres flics. Mais ayant déposé quelque part un peu de dynamite... " (Le Semeur, n 6, 19661967, p. 13 sq.). Rassurez-vous, il s'agissait uniquement de dynamite métaphorique ! 
la fête. Rapidement le "mouvement de Mai» se dogmatisait en querelles de « chapelles» (ce terme vient significativement sous la plume!) révolutionnaires. Mes mouvements d'appartenance : le CRIR et Vive la révolution (mouvement d'anarcho-maoïstes, opposés aux maoïstes orthodoxes de la Gauche Prolétarienne) se dissoudront eux-mêmes en 1971-1972.

Des questions refoulées auparavant devenaient obsédantes dans la mesure où l'échec de l'utopie montrait la nécessité d'un nouveau point de départ. Nous avions voulu, en bons post-marxistes, lier étroitement l'interprétation du monde à l'obligation de le transformer. Mais le «vieux monde » résistait toujours et ses «aliénations» contaminaient les partisans du «nouveau monde ». Il fallait donc chercher à interpréter le monde de façon autonome. Nous avions voulu lutter contre l'idéologie dominante, nous avions nous-mêmes effectué une instrumentalisation idéologique. Bref, il n'était plus possible de mettre d'un côté l'objectivation scientifique liée aux contraintes d'un travail universitaire, de l'autre, la «vraie vie », celle du "guérillero » d'un nouveau genre, où les discours (y compris ceux des " grands intellectuels» comme Lacan ou Foucault) étaient évalués au profit que pouvait en tirer le projet révolutionnaire. Mais mettre la démarche d'objectivation au cœur même de sa pensée était une révolution intellectuelle très douloureuse et qui n'avait rien d'évident. Abandonner l'idée que tout propos était idéologiquement situé et que personne n'était objectif ne signifiait-il pas se normaliser, devenir semblables à ceux que l'on avait combattus?

La peur de (re)devenir un être humain platement normal, de se "ranger ", d'abandonner l'espoir d'une transformation radicale de la société (le réformisme « centriste » était, naturellement, considéré comme l'ennemi) tarauda longtemps, de mille manières, celles et ceux qui avaient grandi, lycéens, dans l'atmosphère de la guerre d'Algérie, puis participé aux contestations aboutissant à « Mai 68 ".

Pour ma part, ce ne furent pas d'abord les Archives qui m'aidèrent à résoudre le problème de l'objectivation. L'étincelle provint d'un article de Maxime Rodinson dans Diogène, "Sociologie marxiste et idéologie marxiste » ${ }^{26}$ puis, suite à cette étude, de plusieurs rencontres avec son auteur. M. Rodinson m'expliquait patiemment que ce n'était pas parce que l'objectivité n'était pas absolue qu'elle n'existait pas. Il me montrait concrètement, exemples à l'appui, la différence entre des textes idéologiques et militants et des textes où l'objectivité était recherchée sinon toujours absolument atteinte. Son propos apparaissait d'autant plus crédible que ses positions courageuses sur le conflit israélo-palestinien empêchaient de le considérer comme un « intellectuel bourgeois ». Il constitua donc un maillon indispensable du tournant opéré, en même temps qu'il devint un ami, en dépit de la différence d'âge. 104.

26. M. Rodinson, «Sociologie marxiste et idéologie marxiste ", Diogène, 64, 1968, p. 70- 
C'est donc du tournant des années 1960 et des années 1970 que date ma véritable prise de contact, suivie d'une progressive intégration. À dire vrai, ce fut une rencontre avec le Groupe de Sociologie des Religions, noyau central de l'équipe des Archives et pas seulement avec la Revue elle-même. Les cours - notamment ceux d'Émile Poulat et de Jean Séguy, suivis avec assiduité jusqu'en 1978 -, les ouvrages, les articles publiés dans d'autres revues étaient indissociables des Archives elles-mêmes. Les Archives constituèrent en quelque sorte le noyau dur d'un ensemble plus vaste.

La revue favorisa d'abord des séances de "rattrapage » et, en plus des nouveaux numéros attendus avec impatience, des numéros déjà publiés furent achetés et lus, cette fois, avec passion. En témoigne les phrases surlignées dans de nombreux articles et recensions d'ouvrages ${ }^{27}$ et deux études d'Émile Poulat littéralement arrachées, sans doute pour pouvoir être plus facilement transportées : significativement la première de ces études traite du passage d'une utilisation polémique des notions de "modernisme " et d'" intégrisme " à un "irénisme critique ${ }^{28}$, ce qui correspondait tout à fait au souhait d'apprendre comment maitriser des instruments permettant une démarche objectivante; la seconde étude analyse "l'ébranlement " du "système d'emprise catholique " ${ }^{29}$, et rencontrait la préoccupation de pouvoir comprendre comment s'effectuait les rapports entre la religion et la modernité.

Ce fut aussi, à ce moment-là, la découverte des œuvres de Max Weber mal connues, même si Rodinson m'avait recommandé la lecture de Le savant et le politique $^{30}$, étape importante dans la légitimation d'une démarche sociologique objectivante : ce qu'indiquait Weber sur les «faits inconfortables » que le marxisme n'avait pas affrontés faisait totalement sens. Cette découverte plus complète commença par la lecture de la recension du livre de Philippe Besnard, Protestantisme et capitalisme, la controverse post-wébérienne ${ }^{31}$ (puis, naturellement de

27. La relecture de ces pages montre que les ASR (puis les ASSR) ont permis aussi une ouverture très forte à la recherche internationale dans les différents domaines (sociologie, histoire mais aussi psychologie religieuse...) et à la critique d'ouvrages de différentes disciplines, y compris parfois théologiques: ainsi les pages 190-192 des ASR, 30, 1970, sont abondamment surlignées : il s'agit de la critique par J. Hutton de l'édition française de l'ouvrage d'H. ConZelmanN (qualifié de "disciple de Bultmann »), Théologie du Nouveau Testament, paru aux éditions du Centurion en 1969. J. H. estimait que ce type d'exégètes se trouvait "dans une situation paradoxale, puisque leur souci "théologique" est en contradiction avec leur mentalité "historique" et se demandait si ce n'est pas le concept même de "théologie du Nouveau Testament" qui doit être mis en question ». Naturellement, un parcours des recensions surlignées montre que ce sont les ouvrages de théorisation sociologique et d'histoire qui me concernaient le plus directement.

28. É. Poulat, " "Modernisme" et "Intégrisme". Du concept polémique à l'irénisme critique », ASR, 27, 1969, p. 3-2.

29. É. Poulat, «Le Catholicisme devant l'ébranlement de son système d'emprise ", ASR, 28, 1969, p. 131-147.

30. M. Weber, Le Savant et le politique, Paris, Plon, 1959.

31. Faite par J. SÉGUY in $A S R, 30,1970$, p. 175 sq. 
l'ouvrage lui-même ${ }^{32}$ ) et se poursuivit par plusieurs lectures de l'article manifeste de Jean Séguy « Max Weber et la sociologie historique de la religion ${ }^{33}$ qui faisait plus qu'introduire à l'étude des œuvres elles-mêmes et notamment à Économie et Société ${ }^{34}$. D'autres approches de Weber se trouvaient également publiées dans les Archives, ce qui permettait de connaître et de comparer les approches et les interprétations ${ }^{35}$ (même si nous préférions celles de notre professeur !). Le fait de construire des types-idéaux, de les considérer comme des instruments opérationnels et des signalements provisoires plausibles, les vérifications et les évaluations d'éléments de la réalité empirique opérées à partir de ces constructions, constituaient le sésame qui permettait de prendre de la distance avec tout substantialisme. Mais Weber ne remplaçait pas Marx comme maître à penser, d'abord parce que Séguy nous en parlait comme d'un ennemi de tout système, ensuite parce qu'au même moment, les Archives publiaient « un texte peu connu » de Durkheim ${ }^{36}$ et y voyait une "admirable invitation à [le] lire ou le relire ${ }^{37}$. Les filiations diverses (y compris celle de Marx), le pluralisme des méthodes et des problématiques, le refus de toute théorie englobante étaient mis en avant et pratiqués.

La fréquentation de l'équipe des Archives permettait, en outre, de revisiter son propre itinéraire et les liens implicites du politique et du religieux que comportait les effervescences « révolutionnaires » des années soixante : les ouvrages d'Henri Desroche et ses articles ${ }^{38} \mathrm{y}$ contribuèrent de façon forte. Un renversement

32. Ce furent à la fois les indications bibliographiques données par les membres du GSR au début de leurs cours et les recensions des Archives qui nous permettaient de savoir ce qu'il fallait absolument lire.

33. J. SÉGUY, "Max Weber et la sociologie historique des religions ", ASR, 33, 1972, p. 71104, J. Séguy nous avait déjà introduits à la pensée de Troeltsch : "Ernst Troeltsch. Ou de l'essence de la religion à la typologie des christianismes ", ASR, 25, 1968, p. 3-11.

34. Rappelons que le début des années 1970 fut marqué par la parution de l'édition française du Judaïsme antique (Paris, Plon, 1970) et du tome 1 d'Économie et société (Paris, Plon, 1971 ) ; on attend toujours la parution de l'édition française du tome 2 !

35. Voir par exemple, S.N. EISENSTADT, "Some Reflections on the Significance of Max Weber's Sociology of Religions for the Analysis of Non-European Modernity ", ASR, 32, 1971, p. 29-52 et B. Nelson, "Droit canon, Protestantisme et "Esprit du capitalisme". À propos de Max Weber ", ASR, 34, 1972, p. 3-23.

36. É. Durkheim, «Le sentiment religieux à l'heure actuelle » (1914), ASR, 27, 1969, p. 73-77, introduit par C. Hamès « Un texte peu connu de Durkheim », idem, p. 71-72.

37. H. Desroche, "Retour à Durkheim ? D’un texte peu connu à quelques thèses méconnues ", ASR, 27, 1969, p. 79. É. POUlAT, « La conception sociale de la religion. Retour à un texte peu connu de Durkheim ", ASR, 30, 1970, p. 87-90.

38. À ceux cités notes 15 et 22, ajoutons «Messianismes et utopies. Notes sur les origines du Socialisme occidental ", ASR, 8, 1959, p. 31-46. " "Dernière instance" et "premier rôle" ", ASR, 23, 1967, p. 153-158 et "Sociologie religieuse et liturgie sociale dans l'œuvre de Charles Fourier ", ASR, 33, 1972, p. 3-32. Le début des années 1970 fut un temps d'intense production pour H. Desroche avec la parution de trois ouvrages : Les Dieux rêvés, Paris, Desclée, 1972 ; L’Homme et ses religions, Paris, Cerf, 1972 et Sociologie de l'espérance, Paris, CalmannLévy, 1973. 
s'était opéré par rapport à la décennie précédente : l'engagement politique était devenu plus circonscrit et l'engagement scientifique plus global. Les Archives nous invitaient à un élargissement au-delà de la sociologie de religions comme le christianisme en ses diverses composantes et le judaïsme (étudié par Doris Bensimon ${ }^{39}$ ): islam, bouddhisme, religions africaines devaient également être objet de l'investigation des sciences sociales ${ }^{40}$. "Sciences sociales » car la sociologie de la religion, telle que la concevait la revue, comportait des passerelles non seulement avec l'histoire mais avec d'autres champs d'études et d'autres sciences de la société : l'analyse du politique et du médical avec François Isambert ${ }^{41}$; la psychologie sociale avec Jean-Pierre Deconchy ${ }^{42}$, la psychanalyse avec Jacques Maître ${ }^{43}$. Le titre Archives de Sociologie des Religions (ASR) fut d'ailleurs modifié en Archives des Sciences Sociales des Religions (ASSR), en 1973, à partir du numéro 35.

Mais, comme il ne s'agit nullement ici de tenir un propos qui fleurerait l'hagiographie, il me faut indiquer le sujet où les Archives me laissèrent un peu insatisfait : celui de la laïcité comprise comme une notion distincte de la sécularisation. Certes, je reçus pas mal de grain à moudre. Mes lectures de rattrapage portèrent notamment sur l'anticléricalisme auquel Émile Poulat avait consacré deux articles importants ${ }^{44}$, ainsi que sur l'intérêt d'Henri Desroche pour le " laïcisme ", dans le cadre de ses études sur les non-conformismes, et qu'il définissait ainsi : "Non-religion pure et simple dont le domaine divers s'accroît de tout ce que la conceptualisation de la langue anglaise aime à nommer la "sécularisation" " ${ }^{45}$.

\footnotetext{
39. Cf. par exemple, D. Bensimon, "Pratique religieuse des Juifs d'Afrique du Nord en France et en Israël », ASR, 26, 1968, p. 81-96.

40. Cf. J.-P. Charnay, "Préalables épistémologiques à une sociologie religieuse de l'islam », ASSR, 37, 1974, p. 79-86 ; Fr. HoutART, "Les fonctions sociales de la symbolique religieuse chez les Bouddhistes à Sri Lanka », idem, p. 23-41; Y. Person, "Pour une histoire des religions africaines ", ASSR, 36, 1973, p.91-101 (on remarquera, dans deux des trois titres, l'intention explicite d'ouvrir de nouveaux champs).

41. F.A. ISAMBERT, «Signification de quelques correspondances empiriques entre comportements politiques et religieux », ASR, 33, 1972, p. 49-70 ; "Les transformations du rituel catholique des mourants" ASSR, 39, 1975, p. 89-100 (dans un numéro publiant les actes d'un colloque du Centre de sociologie du protestantisme de Strasbourg : "L'évolution de l'image de la mort dans la société contemporaine et le discours religieux des Églises »).

42. J.-P. Deconchy, "Psychologie de la mort et psychologie de la religion ", ASR, 33, 1972, p. 105-110 ; "L'Orthodoxie enseignée par renforcement du réglage de l'appartenance ", ASSR, 38, 1974, p. 91-108 (avec des figures mathématiques et des tableaux qui nous impressionnaient beaucoup mais que nous avions parfois de la peine à comprendre !).

43. J. MAître, "Psychanalyse et sociologie religieuses ", ASR, 33, 1972, p. 111-134.

44. É. Poulat, "Une enquête anticléricale de pratique religieuse en Seine-et-Marne (1903) ", ASR, 6, 1958, p. 127-148 ; "Socialisme et anticléricalisme. Une enquête socialiste internationale (1902-1903) », ASR, 10, 1960, p. 109-131.
}

45. H. Desroche, "Approche du non-conformisme français », ASR, 2, 1956, p. 51. 
Nous étions donc renvoyés aux théories anglo-saxonnes de la "sécularisation" auxquelles les Archives n'accordèrent pas de place privilégiée, contrairement à ce qui en a été dit parfois ensuite, mais qu'elles diffusèrent parmi d'autres approches. Et parmi les phrases surlignées, je retrouve celle-ci extraite d'un texte de Peter Berger et Thomas Luckmann: "Sous l'effet du pluralisme, les institutions religieuses se transforment de primaires en secondaires, c'est-à-dire d'institutions contraignantes et monopolisées de la sphère publique en institutions volontaires, concurrentielles de la vie privée ${ }^{46}$. Autrement dit : la pluralisation du champs religieux engendrait sa privatisation sécularisante ${ }^{47}$. Or, dans le cadre de la préparation de ma thèse de doctorat d'État portant sur « Le protestantisme et la laïcisation de la société française » je rencontrais l'absence de véritable pluralisme, la tentative politique (par le système des « cultes reconnus ») d'insuffler un certain pluralisme dans la société française, et son échec, le "conflit des deux France » restant, au bout du compte, prédominant. Les théories de la sécularisation s'avéraient donc insuffisantes pour rendre compte de la situation française, et plus largement du lien des mutations socioreligieuses avec l'instance politique dans les sociétés modernes.

Les Archives exposèrent une piste importante pour saisir ce lien, celle de la « religion civile ", en traduisant et en publiant un texte de Robert N. Bellah, qui allait rapidement devenir un classique ${ }^{48}$. Mais comme d'autres, je le lus alors comme une contribution pour décrypter la situation américaine et ce ne fut que plus tard (toujours les décalages temporels !) que nous lui avons donné une portée plus générale. Comme, après tout, une thèse doit apporter du neuf et que les Archives m'avaient donné le goût de la théorisation, j'entrepris alors de construire, à ma manière, des idéaux types diachroniques, permettant une périodisation à la fois souple et structurée : ce fut l'invention de la notion de seuils de laïcisation. Début d'un parcours qui m'entrainera ultérieurement à changer d'objet d'études, à la création d'une jeune équipe "Histoire et sociologie de la laïcité » en 1990, à la titularisation à une nouvelle direction d'étude à l'EPHE, portant le même nom, l'année suivante et, en 1994-1995, au moment de la fin du Groupe de Sociologie des Religions, à la création, parallèlement à celle du Centre d'études interdisciplinaires des faits religieux (CEIFR, CNRS-EHESS), du Groupe de Sociologie des Religions et de la Laïcité (GSRL, CNRS-EPHE).

Entre temps, l'intégration au Groupe de Sociologie des Religions et à l'équipe des Archives m'avait fait passer du statut de lecteur assidu à celui de collaborateur : à partir de $1973\left(\mathrm{n}^{\circ} 35\right)$, je publiais régulièrement des recensions, en 1980

46. P. Berger, T. Luckmann, "Aspects sociologiques du pluralisme », ASR, 23, 1967, p. 124.

47. Le titre anglais de l'article dont les ASR publiaient une traduction française était: "Secularization and Pluralism ». La disparition de la notion de "sécularisation " dans le titre français est un indice supplémentaire que la dite sécularisation n'était pas la «cup of tea » de l'équipe!

48. R.-N. BelLaH, "La religion civile en Amérique ", ASSR, 35, 1973, p. 7-22. 
paraissait mon premier article, dont le sous-titre ( Tentative d'analyse du pouvoir de contester ») était très significatif de l'itinéraire accompli ${ }^{49}$ et en 1981 j'avais l'honneur de succéder à Émile Poulat comme responsable du Bulletin des ouvrages.

Quelques années auparavant, j'étais devenu directeur d'études à la section des sciences religieuses de l'École Pratique des Hautes Études et «pour la première fois (...) le terme de sociologie faisait son entrée officielle à la $\mathrm{V}^{\mathrm{e}}$ section ${ }^{50}$. La préoccupation de la sociologie à la $\mathrm{V}^{\mathrm{e}}$ section avait été présente bien avant, comme cela a été rappelé à propos d'É.-G. Léonard ${ }^{51}$. Il faut mentionner aussi Gabriel Le Bras, qui y enseigna de 1931 à 1965. Mais aucune direction d'études ne comportait le terme de "sociologie » dans son titre, jusqu'à ce qu'en 1978, la direction d'études « Histoire du protestantisme » devint " Histoire et sociologie du protestantisme " ${ }^{52}$. Or la première moitié des années 1970 s'était trouvée marquée par une distanciation qui avait abouti à la transformation de la $\mathrm{VI}^{\mathrm{e}}$ section de l'École Pratique des Hautes Études en École des Hautes Études en Sciences Sociales. Les turbulences qui en étaient résultées auraient pu engendrer des quant à soi d'institutions et éloigner la section des sciences religieuses d'une référence officielle à la sociologie de la religion ${ }^{53}$. La pertinence scientifique s'avérait donc plus forte que les tensions institutionnelles.

Cela était non seulement heureux mais logique car les Archives ne cachaient pas leur dette à l'égard de la section des sciences religieuses de l'EPHE et, à deux reprises, en 1970 et en 1973, un texte de deux historiens de la Vection, HenriCharles Puech et Paul Vignaux fut republié. Il affirmait ( "généreusement " selon le Comité de rédaction) : "C'est seulement dans la sociologie, qui prend à son tour pour matière les données de la science comparative des religions, que le fait religieux sera entièrement défini et expliqué par la Société, et c'est seulement par la sociologie que la Science des religions pourra être intégrée à l'ensemble des sciences sociales " ${ }^{54}$. Sa date $: 1937$. Texte utopique, peut-être ("entièrement défini et expliqué » !), mais comme les Archives, et Henri Desroche en particulier, nous l'ont appris, si l'utopie ne se réalise jamais telle quelle, elle produit souvent des effets socio-historiques!

49. J. Ваиве́rot, «L'élaboration du document "Église et pouvoirs", tentative d'analyse du pouvoir de contester ", ASSR, 49-1, 1980, p. 7-27.

50. J.-P. Willaime, art. cité, ASSR, 83, 1993, p. 160.

51. Dont, rappelons-le, Jean Séguy a été l'élève.

52. Ce qui fut suivi par deux autres directions d'études et une maîtrise de conférences comportant le terme de «sociologie " dans l'intitulé.

53. Quand j'avais annoncé à D. Robert, dont j'étais l'assistant à l'EPHE, mon intention de suivre les cours de l'équipe des Archives et du GSR, liée à la VI section, il m'avait encouragé à la faire mais, avait-il précisé : "dans votre intérêt, faites-le clandestinement ". Ce dernier terme m'avait beaucoup impressionné.

54. H.-C. Puech, P. Vignaux, "Les Sciences religieuses", in Les Sciences sociales en France. Enseignement et recherches, Paris, Hartmann, 1937. Texte republié par H. DesRoche, J. SÉGuY, éd., Introduction au Sciences Humaines des Religions, Paris, Cujas, 1970, p. 8-35 et citation faite in Le Comité de Rédaction, "À nos lecteurs ", ASSR, 35, 1973, p. 3. 
